\title{
EFEITOS DE UM PROGRAMA DE TREINAMENTO EM CIRCUITO NA COMPOSIÇÃO CORPORAL DE MULHERES ADULTAS
}

\author{
Carin Cássia Benvenutti \\ Universidade Federal de Santa Catarina, Florianópolis, Santa Catarina, Brasil \\ Diego Augusto Santos Silva \\ Universidade Federal de Santa Catarina, Florianópolis, Santa Catarina, Brasil \\ Carla Maria de Liz \\ Universidade do Estado de Santa Catarina, Florianópolis, Santa Catarina, Brasil \\ Edio Luiz Petroski \\ Universidade Federal de Santa Catarina, Florianópolis, Santa Catarina, Brasil
}

\begin{abstract}
Resumo
Este artigo busca verificar os efeitos de um programa de treinamento em circuito na composição corporal de mulheres adultas. Concluíram o experimento $19 \mathrm{mu}-$ lheres, com idade entre 22 e 52 anos, divididas em Grupo Experimental e Grupo Controle. O treinamento teve duração de 12 semanas, composto por 16 estações de exercícios (oito neuromusculares e oito cardiopulmonares), com intensidade de 60 a $85 \%$ da FC máxima, totalizando 30 minutos de atividade. Houve redução na massa gorda e no percentual de gordura no grupo experimental após o período de intervenção. Foi possível concluir, desse modo, que o programa de 30 minutos mostrou-se eficiente para a redução da gordura corporal das investigadas e que pode ser uma ferramenta útil para as pessoas de centros urbanos que não dispõem de tempo para a prática de exercícios físicos.

Palavras-chave: Perda de peso. Mulher. Exercícios. Antropometria.
\end{abstract}

\section{Introdução}

$\mathrm{N}^{\mathrm{s}}$ íveis inadequados de percentual de gordura $(\% \mathrm{G})$, massa de gordura (MG) e massa corporal magra (MCM) estão associados a um maior risco de mortalidade em pessoas de qualquer faixa etária (SARRAFZADEGAN et al., 2009; SHAHRAKI et al., 2008). Nesse sentido, estratégias que atuem nos fatores modificáveis da composição corporal, como alimentação e exercícios físicos, são necessárias para evitar ou diminuir os riscos provenientes do excesso de gordura corporal (SARRAFZADEGAN et al., 2009).

Dentre os exercícios físicos, diferentes formas de treinamento acarretam resultados satisfatórios na composição corporal (TANAKA; 
SWENSEN, 1998; CHTARA et al., 2005; FERNANDEZ et al., 2004; KRAVITZ; VELLA, 2002). No entanto, pesquisadores evidenciaram que o treinamento em circuito mostra-se mais eficiente para a redução da gordura corporal e também para o aumento da MCM, quando comparados aos outros tipos de treinamento (KRAVITZ; VELLA, 2002), isso porque ele combina exercícios cardiopulmonares e neuromusculares, apresentando um volume de intensidade maior. A razão reside no fato de que o treinamento em circuito alterna períodos mais intensos (como ocorre nas estações) e menos intensos (intervalos de recuperação) em uma única sessão. Desse modo, o método consiste na otimização do tempo de execução do exercício, que é dividido em sessões de maior número de repetições, o que estimula o sistema aeróbio e, consequentemente, eleva o gasto energético durante a atividade (SCHNEIDER; MEYER, 2007). Tal alternativa é considerada eficaz para maximizar o gasto energético (KRAVITZ; VELLA, 2002) e evitar a fadiga muscular precoce.

A duração da sessão dos exercícios nas pesquisas com treinamento em circuito gira em torno de 60 minutos (GRANZA et al., 2009; MARX et al., 2001; PULCINELLI; GENTIL, 2002). Todavia, para algumas pessoas a sessão de exercícios físicos demanda muito tempo, se considerados o deslocamento até a academia e o trânsito nas grandes cidades. Como alternativa, surgiram as academias Express, com treinamento de 30 minutos, que é uma maneira de otimizar o tempo de execução do exercício e suprir a necessidade das pessoas que não têm muito tempo (MASCARENHAS et al., 2007).

$\mathrm{O}$ entendimento acerca dos efeitos de programas com treinamento em circuito, sobre a composição corporal de mulheres adultas, é limitado, sobretudo em circuitos de 30 minutos, que vêm ganhando espaço nos centros urbanos. No Brasil, pesquisas com mulheres são escassas, devido à baixa aderência aos programas de contrarresistência, considerando, ainda, que as existentes apresentaram variadas formas de intervenção, não se restringindo a um período de alta e baixa intensidade na mesma sessão, com duração de 30 minutos.

Por isso, o presente estudo tem como objetivo verificar o efeito de um programa de treinamento em circuito de 30 minutos na composição corporal de mulheres adultas. 


\section{Método}

Esta pesquisa seguiu o modelo quase experimental, em que dois grupos escolhidos de forma não aleatória foram avaliados no pré-teste, bem como no pós-teste. O estudo foi aprovado por um Comitê de Ética em Pesquisa com Seres Humanos institucional (protocolo 065/09).

Sujeitos da pesquisa

Participaram do estudo 19 mulheres saudáveis, que não estavam praticando atividade física regular, voluntárias, com idade entre 22 e 52 anos (33 $\pm 8,8$ anos), iniciantes em um programa de treinamento em circuito. As participantes foram convidadas a participar da pesquisa e divididas em dois grupos: Grupo Experimental $(\mathrm{GE})=15$ mulheres e Grupo Controle $(\mathrm{GC})=4$ mulheres. Os critérios impostos no estudo para inclusão das participantes foram: a) não apresentar nenhuma doença crônica ou aguda que pudesse comprometer a composição corporal; b) não participar de nenhum outro programa de atividade física; c) participação mínima em 75\% nas sessões da intervenção.

Ambos os grupos foram submetidos a avaliações antropométricas no início e ao final do período de intervenção. Esse período foi de três meses (12 semanas), no qual o GE realizou o treinamento em circuito, com frequência mínima de três vezes na semana (segundas, quartas e sextas-feiras) e o GC foi orientado a não participar de nenhum programa de atividade física.

Protocolo de treinamento

Após aquecimento de 5 minutos em bicicleta ergométrica, a participante iniciava o treinamento em circuito composto por oito estações de exercícios de contrarresistência, que englobavam os grandes grupos musculares (peitoral, adutores e abdutores da coxa, bíceps, posterior da coxa, grande dorsal, quadríceps e tríceps), ou localizados (glúteo, abdômen e panturrilha), intercalados por oito estações de exercícios cardiopulmonares (step, minicama elástica, corda e saco de boxe). A cada 55 segundos, as mulheres trocavam de estação. Após a passagem nas 16 estações, era dada uma pausa de 15 segundos para aferição da 
FC. O treinamento era finalizado após duas passagens pelas 16 estações.

A verificação da $\mathrm{FC}$ durante o circuito tinha como objetivo controlar a intensidade do treinamento e deveria estar entre $60 \%$ e $85 \%$ da FC máxima de cada participante ( $\mathrm{FCmax}=220$-idade). A mensuração da FC foi ensinada pela pesquisadora durante as avaliações iniciais e aula experimental para conhecimento do circuito, na qual as mulheres foram instruídas a contar o número de batimentos cardíacos por meio da artéria radial, em 15 segundos, e multiplicá-lo por quatro.

A carga $(\mathrm{kg})$ em cada aparelho de contrarresistência e nos exercícios localizados foi determinada nas avaliações iniciais. Para isso, foi levada em consideração a execução correta dos movimentos sem desconforto do sujeito, durante o período de 55 segundos, em cada uma das estações. A cada duas semanas, as participantes eram orientadas a aumentar a carga dos aparelhos, mantendo a correta execução dos movimentos e dentro da FC determinada.

Avaliação antropométrica

As medidas antropométricas de MC, estatura, dobras cutâneas e perímetros corporais foram mensuradas conforme os procedimentos padronizados (PETROSKI, 2007). A MC foi coletada por meio de uma balança digital da marca Toledo ${ }^{\circledR}$, com resolução de 100 gramas e capacidade máxima de $100 \mathrm{~kg}$. A estatura foi verificada por meio do estadiômetro da mesma balança, com escala de resolução de $0,1 \mathrm{~cm}$. A partir dessas medidas calculou-se o IMC (IMC $=\mathrm{MC} /$ Estatura $\left.^{2}\right)$. O perímetro de cintura (PC), abdômen e quadril foram aferidos com fita métrica da marca Cescorf $\AA$, de precisão de $0,1 \mathrm{~mm}$ e calculou-se a relação cintura/quadril (RCQ). As dobras cutâneas (tríceps, subescapular, axilar média, peitoral, abdominal, crista ilíaca e coxa média) foram mensuradas com adipômetro da marca Cescorf®, com precisão de $0,1 \mathrm{~mm}$.

Para identificação do \%G, foi calculada, inicialmente, a densidade corporal (DC) pela fórmula de Jackson, Pollock e Ward (1980) para mulheres adultas: $\mathrm{DC}=1,0970-\left[0,00046971\left(\sum 7\right.\right.$ dobras cutâneas $)+$ 0,00000056 ( $\sum 7$ dobras cutâneas) 2$]$ - [0,00012828 (idade) $]$. Após a identificação da $\mathrm{DC}$, foi estimado o \%G por meio da equação: $\% \mathrm{G}=$ [(4,95/DC) - 4,50] x100 (SIRI, 1961). A MG e a MCM foram calculadas pelas fórmulas: $\mathrm{MG}=\mathrm{MC}(\% \mathrm{G} / 100) ; \mathrm{MCM}=\mathrm{MC}-\mathrm{MG}$. 
Tratamento estatístico

A análise dos dados foi feita de forma descritiva para todas as variáveis, com a determinação das médias e desvio-padrão. A normalidade dos dados foi avaliada pelo teste de Shapiro Wilk. Após testar a normalidade dos dados, foram empregados procedimentos paramétricos para as variáveis $\mathrm{MC}, \mathrm{MCM}, \% \mathrm{G}, \mathrm{PC}$ e RCQ e não paramétricos para a MG e o IMC. Para a comparação intragrupo, no pré e pós-teste, foi utilizado o teste " $t$ " de Student para dados pareados ou o teste de Wilcoxon para dados não paramétricos. Nas comparações intergrupos, foi utilizado o teste " $t$ " de Student para amostras independentes ou o teste de Mann Whitney para dados não paramétricos. Em todas as análises foi aplicado o nível de significância de $5 \%(\mathrm{p}<0,05)$.

\section{Resultados}

Os valores de MC e IMC inicial e final do GE e GC são apresentados na tabela 1 . Não foram detectadas diferenças entre o pré e pósteste para cada grupo. Ao verificar o delta percentual da $\mathrm{MC}$, foram observadas diferenças entre os grupos, indicando que GE diminuiu $1,1 \%$ e o GC aumentou $6,4 \%$.

Tabela 1: Valores expressos em média e desvio-padrão da MC e IMC dos grupos no pré e pós-intervenção

\begin{tabular}{ccccccc}
\hline Grupos & \multicolumn{2}{c}{$\mathrm{MC}(\mathrm{kg})$} & \multicolumn{4}{c}{$\mathrm{IMC}\left(\mathrm{kg} / \mathrm{m}^{2}\right)$} \\
\cline { 2 - 7 } & Pré & Pós & $\Delta \%$ & Pré & Pós & $\Delta \%$ \\
\cline { 2 - 7 } & $\bar{X}(\mathrm{DP})$ & $\bar{X}(\mathrm{DP})$ & & $\bar{X}(\mathrm{DP})$ & $\bar{X}(\mathrm{DP})$ \\
\hline GE & $63,2(8,9)$ & $60,6(9,4)$ & $-4,1(9,1)^{\dagger}$ & $23,2(2,5)$ & $22,9(2,7)$ & $-1,4(3,0)$ \\
& & & & & & \\
GC & $63,3(10,7)$ & $67,3(11,6)$ & $6,4(7,3)$ & $23,4(2,4)$ & $24,8(3,3)$ & $5,9(6,9)$
\end{tabular}

$\mathrm{X}=$ Média; DP $=$ Desvio-Padrão; $\mathrm{MC}=$ Massa Corporal; $\mathrm{IMC}=$ Índice de Massa Corporal; $\mathrm{GE}=$ Grupo Experimental; $\mathrm{GC}=$ Grupo Controle; $\uparrow$ Diferença significativa entre grupos

A tabela 2 mostra os valores de $\mathrm{MG}, \mathrm{MCM}$ e $\% \mathrm{G}$ dos grupos, antes e após o período de intervenção. O GE apresentou uma diminuição na MG de 10,2\%, quando comparado aos valores iniciais, e o GC, um 
aumento de $17,1 \%(\mathrm{p}<0,05)$. Em relação ao $\% \mathrm{G}$, o $\mathrm{GE}$ apresentou uma diminuição de $9,3 \%$, quando comparado aos valores iniciais, entretanto no GC foi verificado um aumento de $9,3 \%(\mathrm{p}<0,05)$.

Tabela 2: Valores expressos em média e desvio-padrão da composição corporal (MG, MCM e \%G) para os grupos Experimental e Controle, pré e pós-treinamento

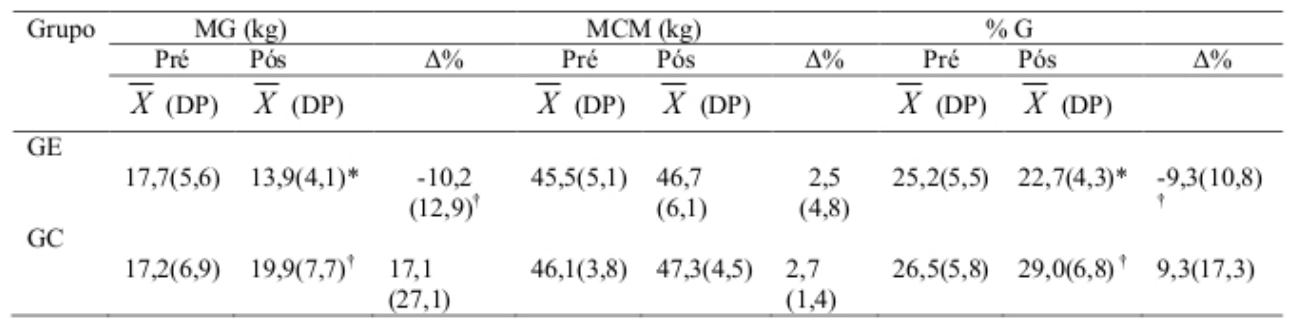

$\mathrm{X}=$ Média; $\mathrm{DP}=$ Desvio-Padrão MG $=$ Massa Gorda MCM = Massa Corporal Magra; $\% \mathrm{G}=$ Percentual de Gordura Corporal; GE $=$ Grupo Experimental; GC = Grupo Controle. * Diferença significativa pré e pós-teste; $\uparrow$ Diferença significativa entre grupos

Os valores referentes ao PC e à RCQ estão expressos na tabela 3 . Na comparação intragrupo e entre grupos, não foi observada diferença significativa para essas variáveis no pré e pós-treinamento.

Tabela 3: Valores expressos em média e desvio-padrão do perímetro da cintura e relação cintura-quadril para os grupos Experimental e Controle, pré e pós-treinamento

\begin{tabular}{ccccccc}
\hline Grupos & \multicolumn{2}{c}{ PC $(\mathrm{cm})$} & \multicolumn{4}{c}{ RCQ } \\
\cline { 2 - 6 } & Pré & Pós & $\Delta \%$ & Pré & Pós & $\Delta \%$ \\
\cline { 2 - 7 } & $\bar{X}(\mathrm{DP})$ & $\bar{X}(\mathrm{DP})$ & & $\bar{X}(\mathrm{DP})$ & $\bar{X}(\mathrm{DP})$ & \\
\hline GE & $69,9(5,7)$ & $69,3(5,9)$ & $-8,0(2,8)$ & $0,71(0,04)$ & $0,73(0,05)$ & $-2,0(2,8)$ \\
& & & & & & \\
GC & $72,6(7,2)$ & $75,8(7,3)$ & $4,4(3,8)$ & $0,71(0,04)$ & $0,73(0,05)$ & $0,7(1,3)$
\end{tabular}

$\mathrm{X}=$ Média; $\mathrm{DP}=$ Desvio-Padrão; $\mathrm{PC}=$ Perímetro da Cintura; $\mathrm{RCQ}=$ Relação Cintura-Quadril; GE = Grupo Experimental; GC = Grupo Controle

\section{Discussão}

As academias Express, que propõem 30 minutos de exercícios físicos diários, adotando o treinamento em circuito, surgiram como uma opção para quem deseja praticar exercícios mas não possui disponibi- 
lidade de tempo. O treinamento em circuito vem se difundindo pelo mundo e investigações acerca da eficácia desse método na composição corporal dos praticantes são fundamentais para a continuidade da sua prescrição, sobretudo em períodos curtos de 30 minutos. As principais conclusões deste estudo residem no fato de que as mulheres que participaram do programa de exercícios físicos alteraram de maneira significativa a composição corporal, o que não foi observado no GC. Tais resultados podem dar subsídios a futuras investigações com esse tipo de treinamento.

As mulheres que realizaram o exercício em circuito diminuíram o $\% \mathrm{G}$ e a $\mathrm{MG}$ ao final da intervenção. Resultados semelhantes foram verificados em outras pesquisas que utilizaram esse tipo de treinamento com um tempo de duração maior para as sessões (MARX et al., 2001; GRANZA et al., 2009). Marx et al. (2001) submeteram 12 mulheres a um treinamento em circuito de seis meses, três vezes na semana, com 60 minutos cada sessão. Os autores observaram que as praticantes diminuíram a gordura subcutânea e abdominal. Granza et al. (2009) submeteram 14 mulheres sedentárias a um programa domiciliar de treinamento em circuito submáximo, com duração de 60 minutos por sessão, três vezes na semana, e evidenciaram reduções no $\% \mathrm{G}$ e $\mathrm{MG}$.

Uma possível justificativa para tais resultados pode estar relacionada ao mecanismo fisiológico que cerca o método do treinamento em circuito. Ele é caracterizado pela variação da intensidade dos exercícios, proporcionando maior ativação e duração do excesso de consumo de oxigênio pós-exercício, o Excess Post-exercise Oxygen Consumption (Epoc). O Epoc é definido como a elevação do consumo de oxigênio para além dos níveis de repouso (BØRSHEIM; BAHR, 2003) e consiste em uma quebra da homeostase após a finalização do exercício. Um período total de Epoc mais longo pressupõe que o metabolismo ficará acima dos níveis de repouso por mais tempo, o que resultará em um aumento no gasto calórico (BØRSHEIM; BAHR, 2003; SHORT; SEDLOCK, 1997).

O treinamento proposto por esta pesquisa não provocou modificações significativas em algumas variáveis. $\mathrm{O}$ aumento da MCM associado a uma diminuição da $\mathrm{MC}$ é importante, pois aumenta a taxa metabólica de repouso, elevando o gasto energético que, por sua vez, pode contribuir para a manutenção do peso em longo prazo (TREVISAN; BURINI, 2007). O IMC, RCQ e o PC são indicadores antropo- 
métricos de risco à saúde e valores elevados dessas variáveis estão associados a eventos cardiovasculares e doenças crônicas degenerativas, tais como diabetes e hipertensão arterial (SARRAFZADEGAN et al., 2009).

O período de intervenção (12 semanas) pode ter sido insuficiente para provocar mudanças nos indicadores antropométricos e na MCM (LEITE; NUNES, 2002; MARX et al., 2001). O estudo feito por Leite e Nunes (2002) analisou os efeitos do treinamento aeróbio e resistido sobre a composição corporal de adultos e verificou mudanças significativas na MCM do GE após oito meses de intervenção. Na investigação desenvolvida por Marx et al. (2001), os praticantes aumentaram a MCM e diminuíram os indicadores antropométricos de gordura corporal.

A duração de cada sessão é outra variável do treinamento em circuito que pode influenciar na eficácia dos resultados da composição corporal (GRANZA et al., 2009; PULCINELLI; GENTIL, 2002; DIAS et al., 2006). Granza et al. (2009) submeteram dois grupos de mulheres sedentárias a um programa de treinamento em circuito, com duração de 60 minutos por sessão. Os autores identificaram que, independentemente da frequência semanal de treino, duas ou três vezes, ocorreram mudanças significantes na $\mathrm{MG}$ e $\% \mathrm{G}$, entretanto, para a MCM, somente quem treinou três vezes na semana obteve resultados satisfatórios. Pulcinelli e Gentil (2002) verificaram que mulheres jovens obtiveram redução significativa no $\% \mathrm{G}$ e aumento na $\mathrm{MCM}$, após 14 semanas de treinamento em circuito, com duração de 50 minutos por sessão, duas vezes por semana. Dias et al. (2006) avaliaram os efeitos de diferentes protocolos de exercício sobre a composição corporal de mulheres jovens e identificaram maior redução na $\mathrm{MC}$ e $\% \mathrm{G}$ no grupo que executava treinamento em circuito, com duração de 40 minutos por sessão.

O presente estudo não foi eficiente para modificar a $\mathrm{MCM}$, no entanto pesquisadores evidenciaram que o desejo feminino em relação ao corpo e sua forma ocorre no sentido de perder peso e reduzir as silhuetas corporais (QUADROS et al., 2010). Dessa forma, o treinamento proposto vai ao encontro do desejo feminino de redução da MG e pode ser empregado para esse fim.

Uma possível justificativa para a não melhora na $\mathrm{MCM}$ das mulheres que participaram do programa de treinamento pode estar relacionada à intensidade do programa, que foi baseada na FC. O controle 
dos exercícios realizados nos grupos musculares localizados ficou prejudicado, tendo em vista que, quando a atividade envolve menos de $1 / 6$ e/ou 1/7 da musculatura, o comportamento da FC não é o melhor parâmetro para o controle da intensidade (HOLLMANN; HETTINGER, 1989). O American College of Sports Medicine (ACSM) recomenda que a intensidade do exercício seja prescrita a partir do teste de uma repetição máxima (1-RM) e que, para adultos saudáveis, os exercícios sejam realizados de $60 \%$ a $80 \%$ de 1 -RM (AMERICAN..., 2010).

Todavia, as atividades em circuito, da maneira como o assunto foi tratado aqui, reflete uma tendência das academias nos dias atuais. Os autores do presente estudo entendem que o ideal seria a prescrição com base no ACSM, entretanto é difícil para os professores que trabalham com as atividades em academia prescreverem os exercícios com base em 1-RM devido à rotatividade e à grande quantidade de alunos/clientes. A proposta da FC seria, portanto, uma forma mais prática e fácil de controle dessa intensidade. Cabe ressaltar, mais uma vez, que o programa apresentado no presente estudo foi eficiente para redução do \%G e da $\mathrm{MG}$, e não da $\mathrm{MCM}$.

Os resultados observados ao final do treinamento poderiam ser melhores se a intervenção fosse combinada com uma orientação nutricional adequada. Pesquisas comprovaram que a atividade física e orientação nutricional, se investigadas como complementares entre si, apresentam resultados mais eficientes para composição corporal, se comparadas às intervenções que as tratam isoladamente (DIAS et al., 2006; SABIA et al., 2004).

A FC de treinamento foi determinada pela fórmula de FC máxima, 220-idade. Entende-se que uma maneira melhor de determinar a intensidade da atividade proposta seria pela fórmula sugerida por Karvonen et al. (1957), $\mathrm{FC}$ de treinamento $=\mathrm{FC}$ basal $+(\mathrm{FC}$ de reserva $\mathrm{x}$ percentual da intensidade de treinamento). Tal método é considerado um dos mais efetivos para determinar as zonas de treinamento, por considerar a FC basal, que representa o condicionamento físico atual. Não obstante, tal fórmula não foi empregada no presente estudo porque as mulheres deveriam aferir a FC basal durante três dias, logo que acordassem. Como muitas das mulheres do presente estudo trabalhavam fora de casa e ainda tinham filhos e afazeres domésticos, elas poderiam esquecer-se de aferir a FC em três dias seguidos. 
Algumas limitações do presente estudo podem ser destacadas, tais como: 1) número reduzido de voluntárias que concluíram a intervenção; 2) a heterogeneidade em relação à idade do grupo investigado (22 a 52 anos), pois a velocidade na modificação da composição corporal é diferente, conforme a faixa etária; 3 ) frequência semanal e período de intervenção, que poderiam ser maiores; 4) o uso de estadiômetro da própria balança é um fator limitante na avaliação da estatura, tendo em vista que não possibilita a correção de vícios posturais, interferindo diretamente na medida.

Como ponto forte do estudo destaca-se a eficiência que o treinamento em circuito teve para a redução dos níveis de gordura corporal da população investigada, o que demonstra que esse tipo de treinamento é uma ferramenta importante para os profissionais da atividade física.

\title{
Conclusão
}

Com base nos resultados levantados no presente estudo, pode-se concluir que o programa de treinamento em circuito de 30 minutos foi eficiente para a redução do \% $\mathrm{G}$ e da $\mathrm{MG}$ das mulheres submetidas ao treinamento. Assim, acredita-se que esse tipo de atividade pode ser útil para as pessoas de centros urbanos que não possuem muito tempo para a prática de exercícios físicos.

Sugere-se, desse modo, que novos estudos sejam feitos, incluindo orientação nutricional ao treinamento em circuito, a fim de que se observem os efeitos dessa combinação.

The effects of a circuit training program on the body composition of women

\begin{abstract}
This study was designed to investigate the effects of a circuit training program on the body composition of adult women. A total of 19 women, aged 22 to 55 years and divided into an experimental and a control group, completed the protocol. The training program lasted 12 weeks and included 16 exercise stations (eight neuromuscular and eight cardiorespiratory exercises) at an intensity of from 60 to $85 \%$ of maximum FC and making a total of 30 minutes of physical activity. The results demonstrated that both fat mass and body fat percentage were reduced in the experimental group after the intervention period. It was concluded that the 30-minute program was effective at reducing the body fat of these women and may be useful for people living in urban centers who do not have time to exercise.
\end{abstract}

Keywords: Weight loss. Women. Exercise. Anthropometry.

Pensar a Prática, Goiânia, v. 15, n. 4, p. 821-1113, out./dez. 2012 
Efectos de un programa de entrenamiento de circuito sobre la composición corporal en mujeres adultas

\section{Resumen}

Este estudio evaluó los efectos de un programa de entrenamiento de circuito sobre la composición corporal en mujeres adultas. Completaron el estudio 19 mujeres, con edades comprendidas entre 22 y 52 años, divididos en grupo experimental y control. El entrenamiento duró 12 semanas y consistió en 16 estaciones de ejercicios (ocho neuromuscular y cardiopulmonar ocho), con una intensidad de 60 a $85 \%$ de FC máxima, con un total de 30 minutos de actividad. Los resultados mostraron que hubo una reducción de la masa grasa y porcentaje de grasa en el GE después del período de intervención. Llegamos a la conclusión de que el programa de 30 minutos fue eficaz para reducir la grasa corporal y pueden ser investigados de una herramienta útil para las personas a los centros urbanos que no tienen tiempo para hacer ejercicio físico.

Palabras clave: Pérdida de Peso. Mujer. Ejercicio. Antropometría.

\section{Referências}

AMERICAN COLLEGE OF SPORTS MEDICINE. ACSM'S Guidelines for exercise testing and prescription. 8th ed. Philadelphia: Lippincott Williams \& Wilkings, 2010.

BØRSHEIM, E.; BAHR, R. Effect of exercise intensity, duration and mode on post-exercise oxygen consumption. Sports Medicine, v. 33, n. 14, p. 1037-60, 2003.

CHTARA, M.; CHAMARI, K.; CHAOUACHI, M.; CHAOUACHI, A.; KOUBAA, D.; FEKI, Y.; MILLET, G. P.; AMRI, M. Effects of intra-session concurrent endurance and strength training sequence on aerobic performance and capacity. British Journal of Sports Medicine, v. 39, n. 8, p. 555-60, 2005.

DIAS, R.; PRESTES, J.; MANZATTO, R.; FERREIRA, C. K. O.; DONATTO, F. F.; FOSCHINI, D.; CAVAGLIERI, C. R. Efeitos de diferentes programas de exercício nos quadros clínicos e funcional de mulheres com excesso de peso. Revista Brasileira de Cineantropometria e Desempenho Humano, v. 8, n. 3, p. 58-65, 2006.

FERNANDEZ, A. C.; MELLO, M. T.; TUFIK, S.; CASTRO, P. M.; FISBERG, M. Influencia do treinamento aeróbio e anaeróbio na massa de gordura corporal de adolescentes obesos. Revista Brasileira de Medicina do Esporte, v. 10, n. 3, p. 152-158, 2004. 
GRANZA, I.; SANTOS, B. V.; MATTOS, W. T. A.; FERNANDES, N. L.; BENDER, E. T. Efeitos do treinamento em circuito personalizado em dois grupos de mulheres sedentárias com idade entre 23 e 49 anos sobre a antropometria e composição corporal durante doze semanas. Revista Brasileira de Prescrição e Fisiologia do Exercício, v. 3, n. 13, p. 04-15, 2009.

HOllmanN, W.; HetTinger, T. Medicina do Esporte. São Paulo, Manole, 1989.

JACKSON, A. S.; POLLOCK, M. L.; WARD, A. Generalized equations for predicting body density of women. Medicine and Science in Sports and Exercise, v. 12, n. 3, p. 175-81, 1980.

KARVONEN, M. J.; KENTALA, E.; MUSTALA, O. The effects of training in heart rate. Annales Medicinae Experimentalis et Biologiae Fenniae, v. 34, n. 3, p. 307-315, 1957.

KRAVITZ, L.; VELLA, C. A. Energy expenditure in differents modes of exercise: ACSM Current Comment, 2002.

LEITE, C. F.; NUNES, V. G. S. Alterações da composição corporal e de VO2máx. em pessoas obesas submetidas a um programa de exercícios aeróbios e resistidos. Revista Brasileira de Ciência do Esporte, v. 24, n. 1, p. 99-116, 2002.

MARX, J. O.; RATAMESS, N. A.; NINDL, B. C.; GOTSHALK, L. A.; VOLEK, J.S.; DOHI, K.; BUSH, J. A.; GÓMEZ, A. L.; MAZZETTI, S. A.; FLECK, S. J.; HÄKKINEN, K.; NEWTON, R. U.; KRAEMER, W. J. Low-volume circuit versus high-volume periodized resistance training in women. Medicine and Science and Sports Exercise, v. 33, n. 4, p. 635-43, 2001.

MASCARENHAS, F.; VIEIRA, C. A.; MARQUES, T. M. A.; BORGES, P. J. A.; SILVA, B. O.; SANTOS, W. B. Acumulação flexível, técnicas de inovação e grande indústria do Fitness: O Caso Curves Brasil. Pensar a Prática, v. 10, n. 2, p. 237-259, jul./dez. 2007.

PETROSKI, E. L. Antropometria: técnicas e padronizações. 3. ed. Blumenau: Nova Letra, 2007. p. 45-56. 
PULCINELLI, A. J.; GENTIL, P. Treinamento com pesos: efeitos na composição corporal de mulheres jovens. Revista da Educação Física, v. 13, n. 2, p. 41-45, 2002.

QUADROS, T. M. B.; GORDIA, A. P.; MARTINS, C. R.; SILVA, D. A. S.; FERRARI, E. P.; PETROSKI, E. L. Imagem corporal em universitários: associação com estado nutricional e sexo. Motriz, v. 16, n. 1, p. 78-85, 2010.

SABIA, R. V.; SANTOS, J. E.; RIBEIRO, R. P. P. Efeito da atividade física associada à orientação nutricional em adolescentes obesos: comparação entre o exercício aeróbio e anaeróbio. Revista Brasileira de Medicina do Esporte, v. 10, n. 5, p. 349-366, 2004.

SARRAFZADEGAN, N.; KELISHADI, R.; DANA, Z.; ESMAILLZADEH, A.; SOLHPOUR, A.; SHIRANI, S.; NADERI, G.; ASGARY, S.; SADRI, G.; KHOSRAVI, A.; BAHONAR, A. Obesity and cardiometabolic risk factors in a representative population of Iranian adolescents and adults in comparison to a Western population: the Isfahan Healthy Heart Programme. Public Health Nutrition, v. 6, p. 110, 2009.

SCHNEIDER, P.; MEYER, F. O Papel do exercício físico na composição corporal e na taxa metabólica basal de meninos adolescentes obesos. Revista Brasileira de Ciência e Movimento, v. 15, n. 1, p. 101-107, 2007.

SHAHRAKI, T.; SHAHRAKI, M.; ROUDBARI, M.; GARGARI, B. $P$. Determination of the leading central obesity index among cardiovascular risk factors in Iranian women. Food and Nutrition Bulletin, v. 29, n. 1, p. $43-8,2008$.

SHORT, K. R.; SEDLOCK, D. A. Excess postexercise oxygen consumption and recovery rate in trained and untrained subjects. Journal of Applied Physiology, v. 83, n. 1, p. 153-9, 1997.

SIRI, W.E. Body composition from fluid spaces and den $\neg 16$. sity: analysis of methods. In: Brozeck J \& Henschel A. (Eds.). Techniques for measuring body composition. Washington, DC: National Academy of Science, 1961. p. 233-244. 
TANAKA, H.; SWENSEN, T. Impact of resistance training on endurance performance. A new form of cross-training? Sports Medicine, v. 25, n. 3 , p. $191-200,1998$.

TREVISAN, M. C.; BURINI, R. C. Metabolismo de repouso de mulheres pós-menopausadas submetidas a programa de treinamento com pesos (hipertrofia). Revista Brasileira de Medicina do Esporte, v. 13, n. 2, p. 133-13, 2007.

Recebido em: 14/03/2011

Revisado em: 13/09/2011

Aprovado em: 27/11/2011

Endereço para correspondência

diegoaugustoss@yahoo.com.br

Diego Augusto Santos Silva

Universidade Federal de Santa Catarina, Centro de Desportos.

Campus Universitário Trindade, Departamento de Educação Física,

Caixa Postal 476, Trindade

88010-970 - Florianopolis, SC - Brasil 